\title{
Acetate supplementation restores chromatin accessibility and promotes tumor cell differentiation under hypoxia
}

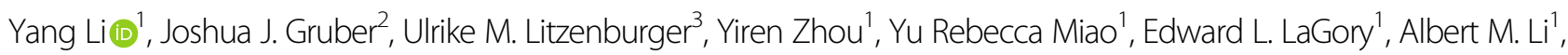
Zhen $\mathrm{Hu}^{4}$, Michaela Yip ${ }^{1}$, Lori S. Hart ${ }^{5}$, John M. Maris ${ }^{5}$, Howard Y. Chang $\mathbb{1}^{3,6}$, Amato J. Giaccia' and Jiangbin Ye (1) ${ }^{1}$

\begin{abstract}
Despite the fact that Otto H. Warburg discovered the Warburg effect almost one hundred years ago, why cancer cells waste most of the glucose carbon as lactate remains an enigma. Warburg proposed a connection between the Warburg effect and cell dedifferentiation. Hypoxia is a common tumor microenvironmental stress that induces the Warburg effect and blocks tumor cell differentiation. The underlying mechanism by which this occurs is poorly understood, and no effective therapeutic strategy has been developed to overcome this resistance to differentiation. Using a neuroblastoma differentiation model, we discovered that hypoxia repressed cell differentiation through reducing cellular acetyl-CoA levels, leading to reduction of global histone acetylation and chromatin accessibility. The metabolic switch triggering this global histone hypoacetylation was the induction of pyruvate dehydrogenase kinases (PDK1 and PDK3). Inhibition of PDKs using dichloroacetate (DCA) restored acetylCoA generation and histone acetylation under hypoxia. Knocking down PDK1 induced neuroblastoma cell differentiation, highlighting the critical role of PDK1 in cell fate control. Importantly, acetate or glycerol triacetate (GTA) supplementation restored differentiation markers expression and neuron differentiation under hypoxia.

Moreover, ATAC-Seq analysis demonstrated that hypoxia treatment significantly reduced chromatin accessibility at RAR/RXR binding sites, which can be restored by acetate supplementation. In addition, hypoxia-induced histone hypermethylation by increasing 2-hydroxyglutarate $(2 \mathrm{HG})$ and reducing a-ketoglutarate (aKG). aKG supplementation reduced histone hypermethylation upon hypoxia, but did not restore histone acetylation or differentiation markers expression. Together, these findings suggest that diverting pyruvate flux away from acetylCoA generation to lactate production is the key mechanism that Warburg effect drives dedifferentiation and tumorigenesis. We propose that combining differentiation therapy with acetate/GTA supplementation might represent an effective therapy against neuroblastoma.
\end{abstract}

\section{Introduction}

The Warburg effect is a metabolic hallmark of all cancer cells, characterized by increased glucose uptake and glycolysis for lactate generation ${ }^{1,2}$. The generation

\footnotetext{
Correspondence: Jiangbin Ye (yej1@stanford.edu)

${ }^{1}$ Department of Radiation Oncology, Stanford University School of Medicine, Stanford, CA 94305, USA

${ }^{2}$ Department of Genetics, Stanford University School of Medicine, Stanford, CA 94305, USA

Full list of author information is available at the end of the article.

Edited by G. Raschellà
}

and excretion of lactate would appear be a waste of carbon backbone and energy that is needed for proliferation. It was proposed by Warburg that the cause and consequence of the Warburg effect were the injury of respiration and cell dedifferentiation, respectively ${ }^{3}$. However, the connection between impaired respiration and cell dedifferentiation has remained unclear due in part to our limited understanding of metabolismdependent epigenetic control. How diverting glycolytic carbon away from the TCA cycle promotes tumorigenesis

\section{(c) The Author(s) 2020}

(c) (i) Open Access This article is licensed under a Creative Commons Attribution 4.0 International License, which permits use, sharing, adaptation, distribution and reproduction cc) in any medium or format, as long as you give appropriate credit to the original author(s) and the source, provide a link to the Creative Commons license, and indicate if changes were made. The images or other third party material in this article are included in the article's Creative Commons license, unless indicated otherwise in a credit line to the material. If material is not included in the article's Creative Commons license and your intended use is not permitted by statutory regulation or exceeds the permitted use, you will need to obtain permission directly from the copyright holder. To view a copy of this license, visit http://creativecommons.org/licenses/by/4.0/. 
remains incompletely understood. As Warburg pointed out, low oxygen-induced injury to mitochondrial respiration is the origin of the Warburg effect. Hypoxia is a common metabolic stress existing in the tumor microenvironment. Previous studies have showed that hypoxia promotes dedifferentiation of neuroblastoma cells toward a neural crest-like phenotype and favors more aggressive features, which in turn resulting in poor clinical outcome $\mathrm{e}^{4-7}$. However, the mechanism by which hypoxia blocks cell differentiation has not been identified.

Neuroblastoma is the most common and deadly pediatric solid tumor, which can arise from neural crest cells that fail to properly exit the cell cycle and differentiate $^{8,9}$. Unlike many other adult cancer types, neuroblastoma has low exonic mutation frequency even in the high-risk group ${ }^{10,11}$. Spontaneous regression often occurs in a subset of neuroblastoma patients ${ }^{12,13}$. The low mutational burden and spontaneous regression that occur in neuroblastoma indicate that reversible epigenetic alterations may play a critical role in regulating neuroblastoma cell differentiation. Retinoic acids induce cell cycle arrest and cell differentiation in neuroblastoma cells $^{14}$, and have been used to treat neuroblastoma as a differentiating agent since the $1980 \mathrm{~s}^{15,16}$. However, the efficacy of RA-based differentiation therapy in neuroblastoma patients is less promising when compared to the treatment outcome of acute promyelocytic leukemia patients $^{17,18}$, especially in high-risk neuroblastoma ${ }^{19}$. The precise mechanism of resistance to differentiation therapy is still not fully understood.

We hypothesize that altered cellular metabolism remodels the epigenetic landscape to block neuroblastoma cell differentiation under hypoxia. Confirming this hypothesis, we reported here that hypoxia suppressed the RA-induced neuroblastoma cell differentiation and the expression of differentiation markers. Neuroblastoma cells exposed to hypoxia exhibited enhanced glycolysis and impaired oxidative phosphorylation, resulting in a significant decrease in both acetyl$\mathrm{CoA}$ and histone acetylation levels. These metabolic changes were associated with hypoxic induction of pyruvate dehydrogenase kinases (PDK1 and PDK3). Inhibition of PDKs by dichloroacetate (DCA) restored histone acetylation under hypoxia. Importantly, acetate supplementation restored histone acetylation, chromatin accessibility, neuron differentiation markers expression and neuron differentiation morphology. Together, these findings suggest that (1) combining RA-based differentiation therapy and acetate supplementation represents a potentially effective therapeutic strategy for neuroblastoma treatment; (2) diverting pyruvate away from acetyl-CoA generation is a key mechanism by which the Warburg effect blocks cell differentiation to promote tumorigenesis.

\section{Results}

\section{Hypoxia represses RA-induced neuron differentiation markers expression}

To test Warburg's hypothesis that injury of respiration causes cell dedifferentiation, two neuroblastoma cell lines, CHP134 and SMS-KCNR, were treated with $10 \mu \mathrm{M} 13-$ cis-retinoic acid (RA) under normoxia $\left(21 \% \mathrm{O}_{2}\right)$ or hypoxia $\left(0.5 \% \mathrm{O}_{2}\right)$. After $48 \mathrm{~h}$ under normoxia, RA treatment induced obvious cell morphological changes to a mature neuronal-like phenotype characterized by neurite outgrowth, while hypoxia partially diminished nerite outgrowth and the differentiation morphology (Fig. 1a, b). Thus, these data indicate CHP134 and SMS-KCNR neuroblastoma cells are appropriate models to investigate the mechanism of differentiation resistance under hypoxia.

Differentiation is determined by expressing specific differentiation markers. To identify neuron-specific differentiation markers that are induced by RA treatment but suppressed by hypoxia, we performed RNA-Seq analysis to determine transcripts abundance after $24 \mathrm{~h}$ RA or DMSO treatment under normoxia or hypoxia. Pathway analysis of RA-upregulated genes indicated the enrichment of NGF signaling, transmission across of chemical synapses and neuronal system genes by RA treatment (Supplemental Fig. S1). We hypothesize that genes that are involved in neuroblastoma differentiation will be induced by RA treatment under normoxia but not under hypoxia. Following this criterion, we identified a group of genes as potential neuron-specific markers (Fig. 1c). Two representative differentiation markers are nerve growth factor receptor (NGFR) and synuclein gamma (SNCG) among others (Fig. 1d). It was reported that NGFR was downregulated by MYCN amplification to maintain an undifferentiated and more aggressive phenotype $^{20}$. SNCG is traditionally characterized as a neuronal marker which is highly expressed in peripheral sensory neurons ${ }^{21}$. Using a public dataset from the R2 Genomics Analysis and Visualization Platform (http://r2. amc.nl $)^{22}$, we found that higher expression of NGFR and SNCG associated with a better overall survival rate (Fig. 1e). Moreover, the expression of NGFR and SNCG was negatively correlated with the expression of hypoxia marker PDK1 (Fig. 1f). Altogether, our results indicate that the expression of these neuronal differentiation markers is repressed under hypoxia, and the hypoxic tumor microenvironment may present a challenge to the efficacy of differentiation therapy in the treatment of neuroblastoma.

\section{Hypoxia induces histone hypoacetylation by reducing acetyl-CoA and citrate generation}

$\mathrm{RA}$ is the ligand of nuclear retinoid and rexinoid receptors (RAR and RXR). Activation of RA-dependent 


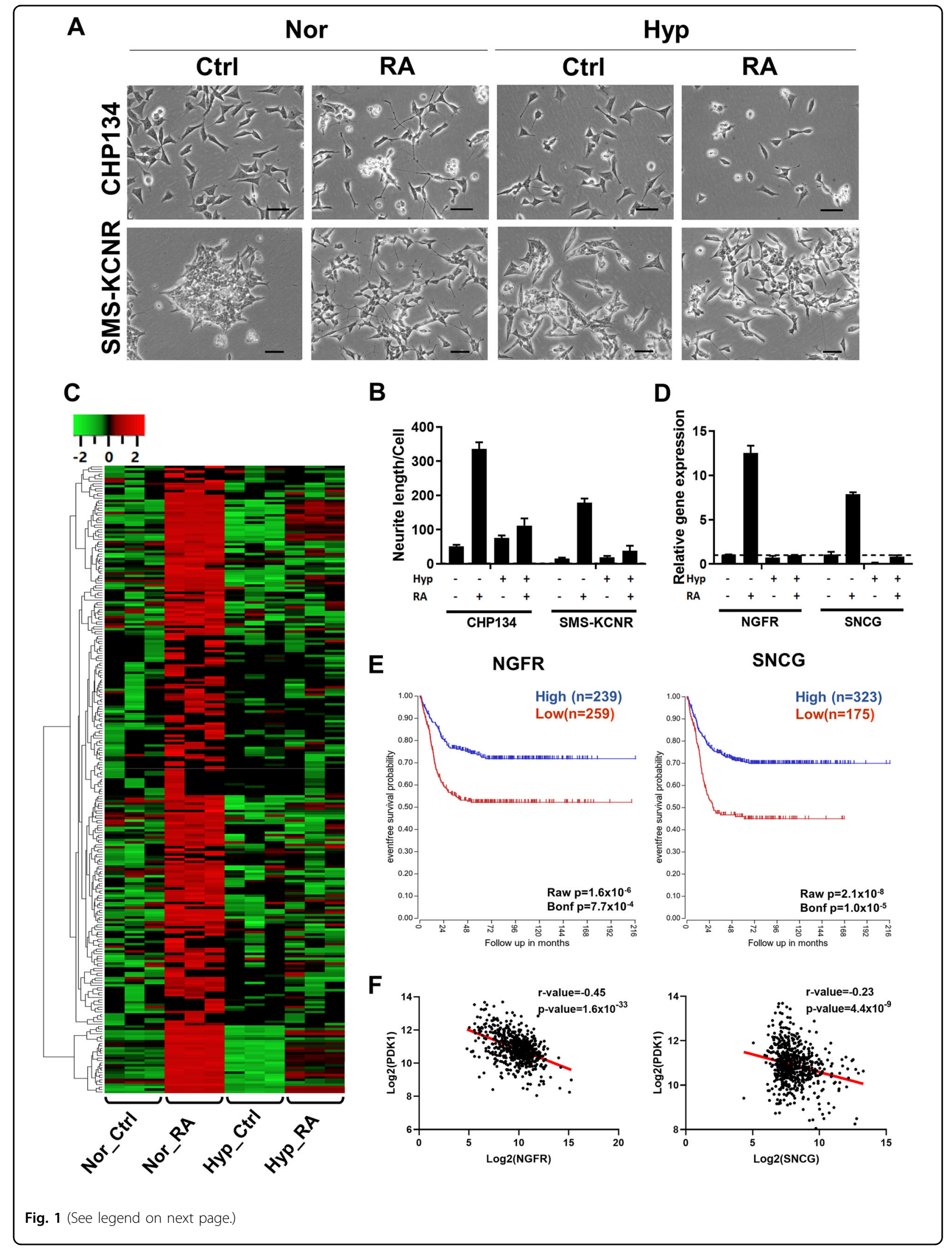


(see figure on previous page)

Fig. 1 Hypoxia disrupts RA-induced differentiation by suppressing the expression of differentiation markers in neuroblastoma cells. a CHP134 and SMS-KCNR cells were treated with $10 \mu \mathrm{M}$ RA for $48 \mathrm{~h}$ under normoxia or hypoxia. Representative image from each treatment group showed the morphologic changes. Scale bar: $50 \mu \mathrm{m}$. b Quantification of neurite outgrowth in (a) with NeuronJ, a plugin in the ImageJ package. c Heatmap and hierarchical clustering of the potential differentiation markers that were induced by RA treatment but repressed by hypoxia treatment. Gene expression levels were determined by RNA-Seq $(n=3)$. d NGFR and SNCG expression levels in CHP134 cells treated with $10 \mu \mathrm{M}$ RA or DMSO under normoxia or hypoxia $(n=3)$. e Overall survival of neuroblastoma patients grouped by NGFR or SNCG expression level. f The negative correlation between the expression of differentiation markers and hypoxic marker PDK1 in neuroblastoma samples. (The analyses in $\mathbf{e}, \mathbf{f}$ were performed on publicly available dataset from R2: Genomic Analysis and Visualization Platform (http://r2.amc.nl).

transcriptional signaling requires histone acetyltransferases (HATs) to acetylate histone, leading to chromatin remodeling over target gene promoters ${ }^{23,24}$. Given that HATs activity is required for RA-induced differentiation, we next explored whether hypoxia regulated chromatin accessibility through changing histone acetylation status. We observed that acetylation of histone $\mathrm{H} 3 \mathrm{~K} 9, \mathrm{H} 3 \mathrm{~K} 27$, and total $\mathrm{H} 3$ acetylation were significantly decreased under hypoxia in both CHP134 and SMSKCNR cells (Fig. 2a), indicating that histone acetylation levels were controlled by oxygen availability. In mouse embryonic fibroblasts (MEFs), a non-tumorigenic cell line, we observed hypoxia treatment increased histone acetylation at $6 \mathrm{~h}$, but significantly decreased histone acetylation at $24 \mathrm{~h}$ (Supplemental Fig. S2A). HATs require acetyl-CoA as substrate in the acetylation reaction. Cytosolic pyruvate can be transported into mitochondria and converted to acetyl-CoA through pyruvate dehydrogenase $(\mathrm{PDH})$. Mitochondrial acetyl-CoA is then combined with oxaloacetate to produce citrate in a reaction that is catalyzed by citrate synthase. Importantly, acetyl-CoA cannot cross the mitochondrial membrane, while citrate can be transported across mitochondrial membrane, meaning that mitochondrial acetyl-CoA must first be converted to citrate before it can contribute to the cytosolic pool of acetyl-CoA. In cytosol, citrate is the major source of acetyl-CoA production through a reaction catalyzed by ATP citrate lyase $(\mathrm{ACLY})^{25}$. To investigate whether hypoxia regulates cellular acetyl-CoA and citrate levels, CHP134 cells were cultured under normoxia or hypoxia, and their intracellular metabolites were extracted and profiled by liquid chromatography-mass spectrometry (LC-MS). Notably, a significant increase in glycolytic intermediates and lactate was observed in hypoxic cells, confirming the enhanced Warburg effect under hypoxia (Supplemental Fig. S2B). We also found that both acetyl-CoA and citrate levels decreased after $24 \mathrm{~h}$ of hypoxia treatment, suggesting that hypoxia treatment downregulated total intracellular acetyl-CoA and citrate (Fig. 2b, c). Other TCA intermediates including $\alpha$ ketoglutarate, succinate, malate, and fumarate also decreased after $24 \mathrm{~h}$ of hypoxia treatment (Supplemental Fig. S2C).
To determine whether the reduction of acetyl-CoA and citrate under hypoxia is caused by decreased pyruvate flux entering the TCA cycle, we performed an isotope tracing study using $\mathrm{U}^{13} \mathrm{C}$-Glucose as a tracer and profiled the mass isotopomer distribution of the downstream metabolites by high-resolution LC-MS (Fig. 2d). A mass isotopomer distribution is a profile containing the relative abundances of each successive mass isotopomer (i.e., $\mathrm{M}$ $+0, M+1, M+2$, and so on). After correction of natural isotope distribution, the specific distribution of stable isotope labeled precursors into metabolic products can be used to represent the metabolic dynamics/flux through the metabolic networks. Consistent with targeted metabolomics analysis, hypoxia treatment resulted in a lower labeling fraction of TCA intermediates including malate $[\mathrm{M}+2]$, fumarate $[\mathrm{M}+2]$, and citrate $[\mathrm{M}+2]$, but higher labeling fraction of lactate $[\mathrm{M}+3]$ (Fig. 2e, f, Supplemental Fig. S2D and E). Acetyl-CoA can be labeled by $\mathrm{U}_{-}{ }^{13} \mathrm{C}-$ Glucose at the acetyl group, ribose group or both, which reflected by mass shifts of $[M+2],[M+5]$ or $[M+7]$ respectively. We found the labeling fraction of acetyl-CoA $[\mathrm{M}+2]$ and $[\mathrm{M}+7]$ were both decreased under hypoxia (Fig. 2g), indicating that carbon flux into the acetyl-CoA pool through pyruvate was reduced. Altogether, these results suggest that reduction of citrate and acetyl-CoA levels under hypoxia is due to decreased pyruvate flux entering the TCA cycle.

\section{Inhibition of PDKs activity restores pyruvate flux into TCA cycle and histone acetylation}

We next explored the molecular mechanism by which hypoxia reduces acetyl-CoA generation. The pyruvate dehydrogenase $(\mathrm{PDH})$ complex is the key enzyme that controls the pyruvate entry into the TCA cycle by converting pyruvate to acetyl-CoA. It has been previously reported that hypoxia-inducible factor-1 (HIF-1) induces the expression of PDK1 and PDK3 which then phosphorylate and inhibit $\mathrm{PDH}$ to reduce pyruvate entry into the TCA cycle ${ }^{26-28}$. This reduces reactive oxygen species levels and protects cells under stress condition. Thus, PDK is critical in regulating oxidative phosphorylation and the intracellular levels of TCA intermediates including citrate, which could potentially regulates histone 


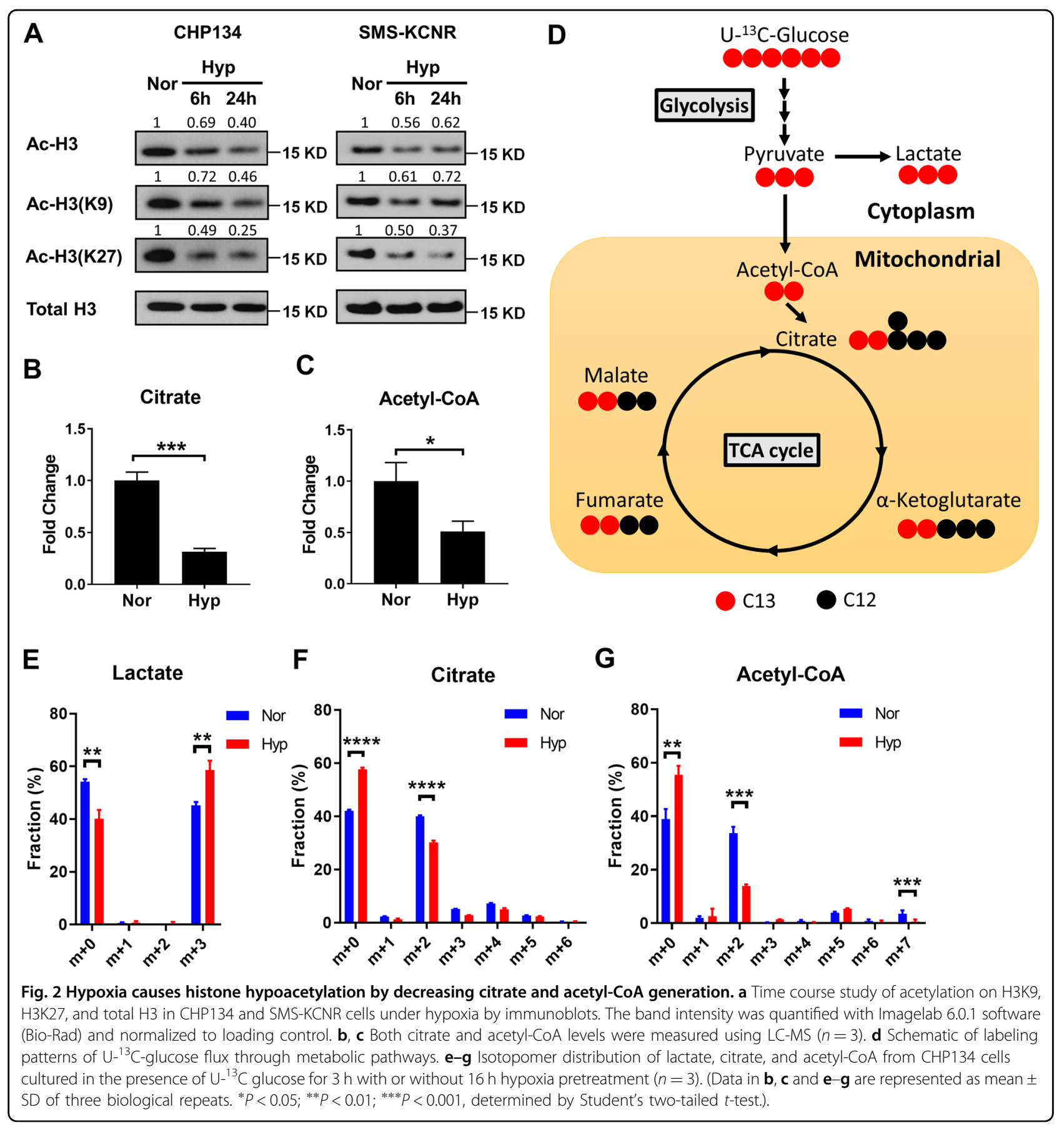

acetylation status ${ }^{29}$. PDK1 and PDK3 induction under hypoxia in CHP134 cells was validated by our RNA-Seq data (Supplemental Fig. S2F). Under hypoxia, PDK1 protein level was significantly increased, associated with elevated PDH phosphorylation (Fig. 3a). To determine whether PDK induction under hypoxia is responsible for reduction of histone acetylation, we tested whether dichloroacetate (DCA), an inhibitor of PDKs, could restore histone acetylation by promoting acetyl-CoA production. Our results showed that DCA treatment inhibited PDK1 activity and decreased PDH phosphorylation under hypoxia (Fig. 3a). While DCA treatment slightly increased acetylation of $\mathrm{H} 3 \mathrm{~K} 9$ and total acetyl-H3 under normoxia, the acetylation restoration effect was much more pronounced under hypoxia. In particular, DCA treatment restored H3K27 acetylation in a dosedependent manner under hypoxia (Fig. 3a). In addition, isotope tracing study further confirmed that DCA treatment under hypoxia increased labeling fraction of acetylCoA $[M+2]$ and citrate $[M+2]$, indicating that PDH 
A

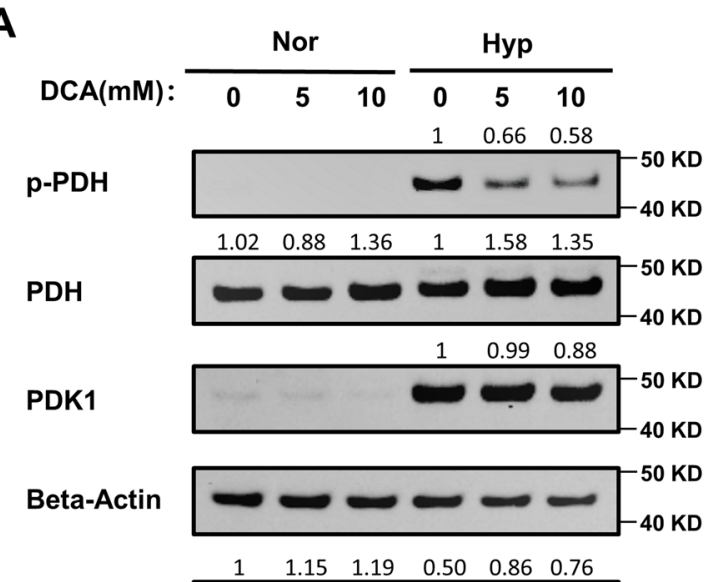

Ac-H3

Ac-H3(K9)

Ac-H3(K27)

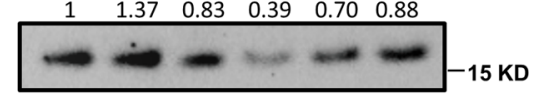

Total H3

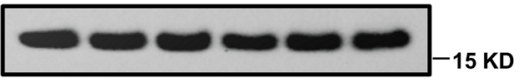

B

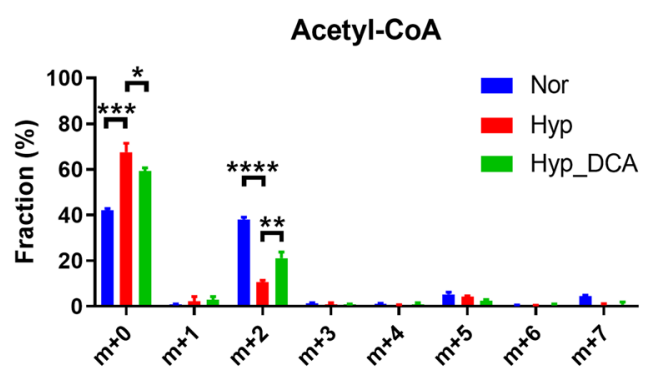

C

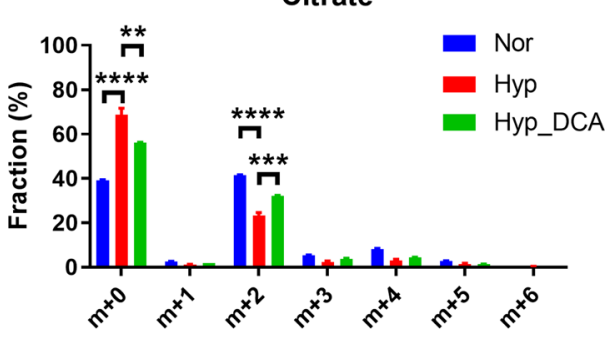

D
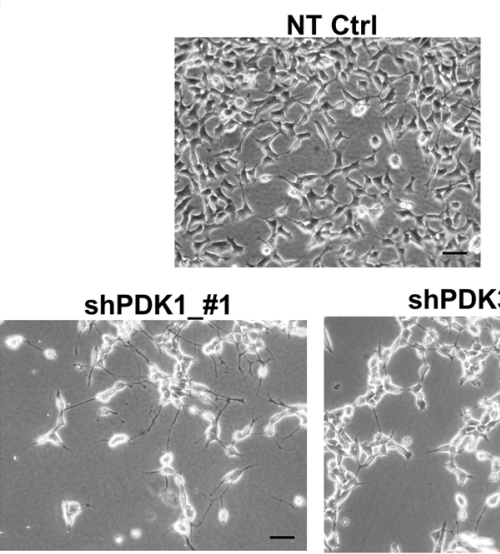

shPDK1 \#2

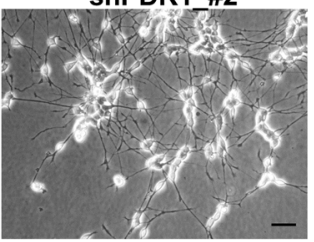

shPDK1 \#3

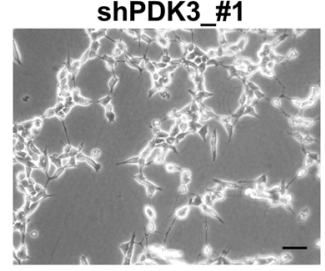

shPDK3 \#2

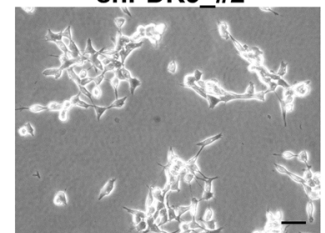

shPDK3_\#3
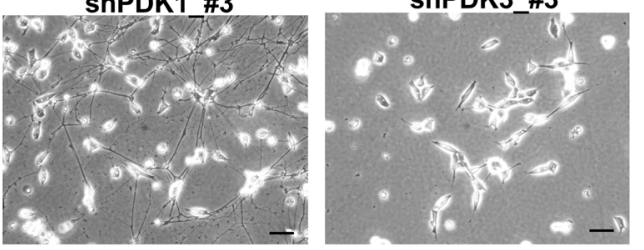

E

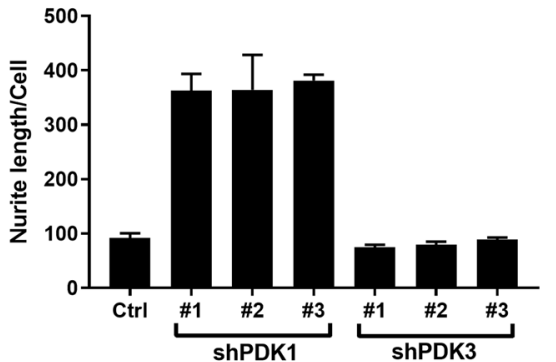

Fig. 3 DCA restores pyruvate flux into TCA cycle and histone acetylation. a Immunoblots of p-PDH, total PDH, PDK1, beta-Actin, acetylation on H3K9, H3K27 and total H3 under normoxia or hypoxia treated with DMSO, $5 \mathrm{mM}$ or $10 \mathrm{mM} \mathrm{DCA.} \mathrm{b,} \mathrm{c} \mathrm{Isotopomer} \mathrm{distribution} \mathrm{of} \mathrm{citrate} \mathrm{and} \mathrm{acetyl-}$ CoA from CHP134 cells cultured in the presence of $\mathrm{U}-{ }^{13} \mathrm{C}$ glucose for $3 \mathrm{~h}$ under normoxia, hypoxia, or hypoxia with $5 \mathrm{mM}$ DCA treatment. (Data in $\mathbf{b}, \mathbf{c}$ are represented as mean \pm SD of three biological repeats. ${ }^{*} P<0.05$; ${ }^{* *} P<0.01$; ${ }^{* *} P<0.001$, determined by Student's two-tailed $t$-test.). $\mathbf{d}$ CHP134 cells were infected with lentivirus expressing shRNA targeting PDK1 or PDK3 (three independent vectors). After puromycin selection, representative images from each pool population were shown. e Quantification of neurite outgrowth in (d).

activity had been partially rescued (Fig. 3b, c). Taken together, our results indicate that PDK inhibition restores histone acetylation under hypoxia by increasing the flux of pyruvate entry into the TCA cycle through the PDH complex. Next, we tested whether knocking down PDK1 or PDK3 could induce cell differentiation. Intriguingly, all 
three shRNA vectors targeting PDK1 caused significant cell death in CHP134 cells after puromycin selection (data not shown), the cells survived displayed neuron differentiation morphology and stopped proliferating (Fig. 3d, e). In contrast, all three shRNA vectors targeting PDK3 only reduced cell proliferation but did not induce neuron differentiation morphology (Fig. 3d, e), suggesting a critical role of PDK1 in regulating neuroblastoma cell differentiation.

\section{Acetate supplementation restores histone acetylation and promotes RA-induced differentiation}

Acetate can supplement the cellular acetyl-CoA pool though Acetyl-CoA synthetases (ACSS1/2/3). ACSS1 and ACSS3 localize to mitochondria, converting acetate to acetyl-CoA for ATP production, while ACSS2 localizes to cytoplasm and contributes to the cytosolic and nuclear acetyl-CoA pool ${ }^{30,31}$. Interestingly, it was shown that ACSS2 was necessary for neuronal genes expression and memory formation ${ }^{32}$. Since we observed that hypoxia prevented RA-induced differentiation of neuroblastoma cells and this lack of differentiation was associated with histone $\mathrm{H} 3$ hypoacetylation and a depletion of acetyl$\mathrm{CoA}$, we reasoned that replenishing acetyl-CoA level with acetate supplementation would restore histone acetylation and RA-induced differentiation under hypoxia. Consistent to a previous report that hypoxia induces $\mathrm{ACSS}_{2}{ }^{33}$, our RNA-Seq data indicated that hypoxia specifically induced the expression of cytosolic ACSS2, but not mitochondrial ACSS1 and ACSS3 (Supplemental Fig. S3A), giving further support for the potential of acetate supplementation to increase acetyl-CoA levels under hypoxia. To test this hypothesis, CHP134 cells were supplemented with $5 \mathrm{mM}$ acetate, 0.5 or $2 \mathrm{mM}$ glycerol triacetate (GTA). GTA is a short-chain triglyceride that can release three molecular equivalents of acetate per molecule of GTA and has been proven as an effective acetate precursor. FDA has approved GTA as a food additive for infants with Canavan disease, since GTA treatment had no detectable toxicity even when using high dose. In our study, both acetate and GTA treatment restored acetylation on $\mathrm{H} 3 \mathrm{~K} 9, \mathrm{H} 3 \mathrm{~K} 27$, and total $\mathrm{H} 3$ under hypoxia (Fig. 4a). To explore how acetate is utilized by the cells, we performed a tracing study with $\mathrm{U}_{-}{ }^{13} \mathrm{C}$-acetate as a tracer. We observed the percentage of ${ }^{13} \mathrm{C}$ labeled citrate $[\mathrm{M}+2]$, malate $[\mathrm{M}+2]$ and fumarate $[\mathrm{M}+2]$ was similar between normoxia and hypoxia. However, hypoxia treatment led to a higher fraction of ${ }^{13} \mathrm{C}$ labeled acetyl$\mathrm{CoA}[\mathrm{M}+2]$ than normoxia (Fig. 4b), indicating acetate supplementation can promote cellular acetyl-CoA production under hypoxia.

Next, we investigated whether acetate supplementation could restore the expression of differentiation markers under hypoxia. CHP134 cells were cultured under normoxia or hypoxia for $16 \mathrm{~h}$, then RA and/or acetate/ GTA were added to each group to determine the effect of acetate supplementation on RA-induced cell differentiation. Supporting our hypothesis that acetate supplementation could promote differentiation therapy, the results showed that acetate or GTA treatment significantly restored the expression of NGFR and SNCG under hypoxia in both CHP134 and SMS-KCNR cells (Fig. 4c, d, Supplemental Fig. S3B). The regulatory model of histone acetylation and cell differentiation under hypoxia was illustrated in Fig. 4e. Acetate supplementation restores cellular acetyl-CoA pool and histone acetylation under hypoxia, which in turn increases chromatin accessibility and promotes transcriptions of RA response genes.

Additionally, GTA supplementation restored the RAinduced neuron-like morphological changes in both CHP134 and SMS-KCNR cells under hypoxia (Fig. 5a, c, Supplemental Fig. S3C). Neuroblastoma differentiation was further confirmed by immunofluorescence staining against neuron specific $\beta$-tubulin III (Tuj1) and microtubule associated protein 2 (MAP2). RA treatment induced neuronal morphological changes, demonstrated by increased $\beta$-tubulin III/MAP2 positive neurites under normoxia, which was partially suppressed under hypoxia. Combination of RA and acetate treatment could restore neuronal differentiation under hypoxia (Fig. $5 \mathrm{~b}$ and Supplemental Fig. S4). Moreover, combination of RA and GTA re-sensitized CHP134 cells to RA-induced proliferation arrest under hypoxia (Fig. 5d and Supplemental Fig. S3D).

\section{ATAC-Seq analysis reveals that acetate supplementation restores chromatin accessibility at RAR/RXR binding sites upon hypoxia}

We employed ATAC-Seq to investigate the effect of hypoxia and acetate supplementation on chromatin accessibility. Our data indicated hypoxia treatment resulted in a more condensed chromatin structure, while acetate partially restored chromatin accessibility (Fig. 6a, b). Further analysis of genes with RAR/RXR binding sites showed decreased chromatin accessibility of these loci under hypoxia and increased chromatin accessibility by acetate treatment (Fig. 6c, d). Interestingly, pathway analysis demonstrated the genes whose chromatin accessibility was decreased under hypoxia and restored by acetate supplementation were enriched in development biology, axon guidance, signaling by NGF and p75 neurotrophin receptor (NTR) (Fig. 6e). The track of one representative neuron differentiation marker SNCG was shown in Fig. 6f. At upstream of SNCG gene, we identified a peak whose coverage was reduced under hypoxia but restored upon acetate supplementation. 


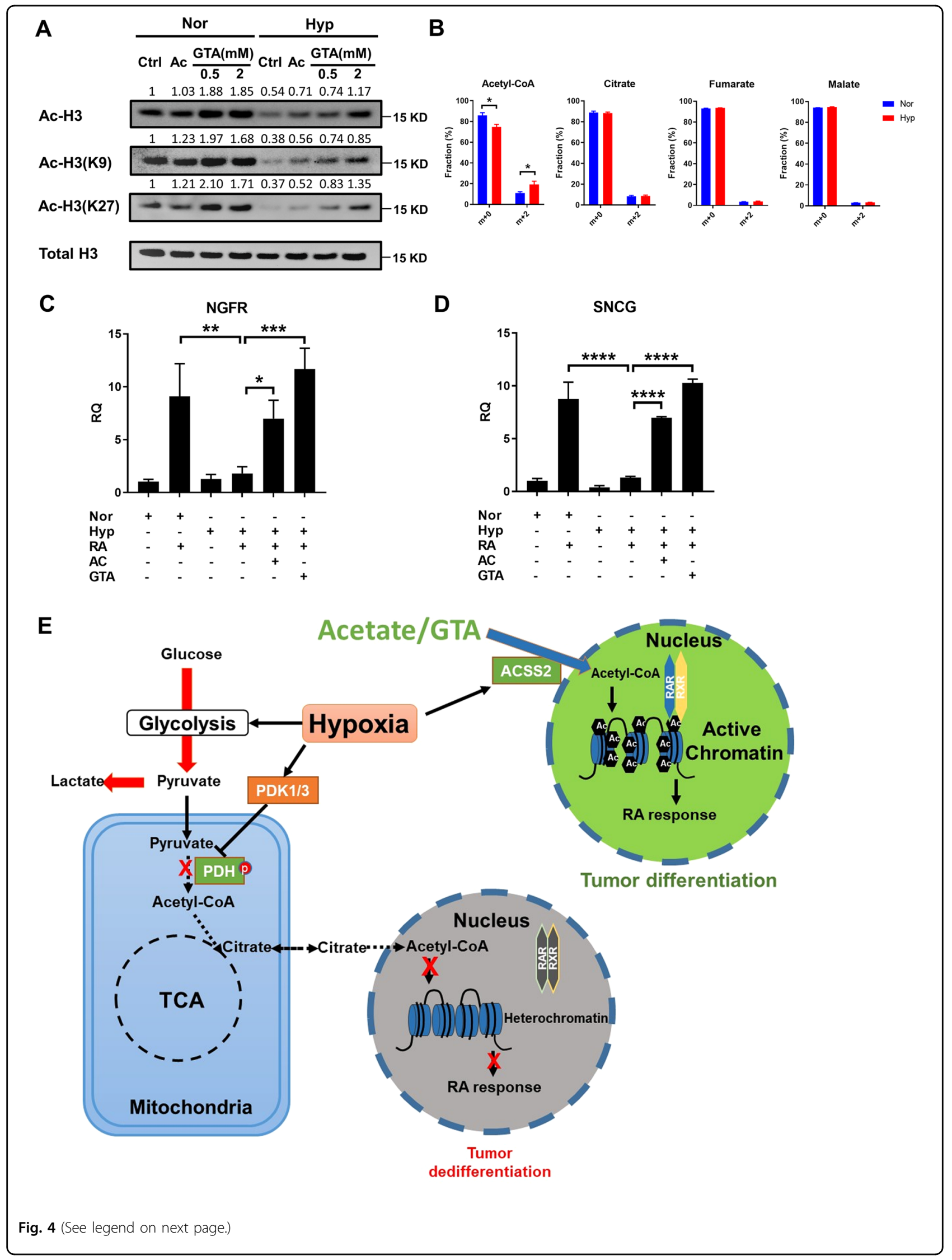


(see figure on previous page)

Fig. 4 Acetate supplementation increases the expression of differentiation markers by promoting histone acetylation. a Immunoblots of acetylation on $\mathrm{H} 3 \mathrm{~K} 9, \mathrm{H} 3 \mathrm{~K} 27$, and total $\mathrm{H3}$ under normoxia or hypoxia treated with vehicle control, $5 \mathrm{mM}$ acetate, $0.5 \mathrm{mM}$ GTA or $2 \mathrm{mM}$ GTA. b Isotopomer distribution of acetyl-CoA, citrate, fumarate, and malate from CHP134 cells cultured in the presence of U- ${ }^{13} \mathrm{C}$ acetate for $3 \mathrm{~h}$ with or without $16 \mathrm{~h}$ hypoxia pretreatment. c, d qPCR analysis for SNCG and NGFR expression in CHP134 cells treated with DMSO, $10 \mu \mathrm{M}$ RA alone, or $10 \mu \mathrm{M}$ RA combined with $5 \mathrm{mM}$ acetate or $2 \mathrm{mM}$ GTA for $16 \mathrm{~h}$ under normoxia or hypoxia. e Model of histone acetylation and cell differentiation regulation under hypoxia. (Data in $\mathbf{b}$ represent mean \pm SD of three biological repeats. Data in $\mathbf{c}$, $\mathbf{d}$ are represented as mean \pm SD of triplicate PCR reactions; a representative of two independent experiments is shown. ${ }^{*} P<0.05$; ${ }^{*} P<0.01$; ${ }^{*}{ }^{*} P<0.001,{ }^{* * *} P<0.0001$, determined by Student's two-tailed t-test.).

In vivo anti-tumor effect of combination of RA and acetate supplementation

Next, we investigated whether acetate supplementation can improve the efficacy of RA in vivo. CHP134 cells were implanted to the flanks of NSG mice and allowed to grow for 3 weeks to reach a volume of $\sim 150 \mathrm{~mm}^{3}$. Then, mice were randomized in four treatment groups: DMSO, RA, GTA or RA + GTA. After 23 days' treatment, RA treatment alone didn't show anti-tumor effect (Fig. 7a, b, Supplemental Fig. S5). Combination of GTA and RA resulted in a slight decrease of average tumor weight compared to RA treatment group. However, the $p$-value did not reach statistical significance $(p=0.106)$, possibly due to the large variation of tumor weight in each group and small sample size (Fig. 7b). The mice body weight in GTA + RA group slightly decreased when comparing to control group (Fig. 7c), and one mouse in GTA + RA group died during the study. These observations suggest that combination of RA and GTA treatment might have a potential toxicity in mice. Pharmacokinetic study showed a relative short half-life of RA. The blood RA concentration reach the peak at $30 \mathrm{~min}$ after i.p. injection and maintained a concentration above $10 \mathrm{uM}$ for around $2 \mathrm{~h}$. At $6 \mathrm{~h}$ after injection, the blood RA concentration reduced to the basal level (Fig. 7d). LC-MS analysis of the plasma samples from control and GTA treatment group indicated a slight increase of plasma acetate concentration by oral GTA administration, though the p-value did not reach statistical significance (Fig. 7e). One possible explanation is that GTA-derived acetate was primarily trapped by liver due to the high activity of $\mathrm{ACSSs}^{34}$.

\section{a-ketoglutarate reduces histone hypermethylation under hypoxia, but cannot restore histone acetylation or differentiation marker expression}

A gain-of-function point mutation on cytoplasmic isocitrate dehydrogenase (IDH1) or mitochondrial isocitrate dehydrogenase (IDH2) grants the mutant enzyme the ability to generate D-2-hydroxyglutarate (D2HG) ${ }^{35}$. Since D2HG has a similar chemical structure as $\alpha$-ketoglutarate $(\alpha K G)$, it can inhibit $\alpha$ KG-dependent enzymes, including TET enzymes and Jumonji domain-containing histone demethylases (JMJDs) ${ }^{36,37}$, leading to DNA and histone hypermethylation that block cellular differentiation.
Recently, it was reported that tumor cells generated the Lenantiomer of 2-hydroxyglutarate (L2HG) under hypoxic conditions. L2HG also inhibits $\alpha-K G$ dependent enzymes including JMJDs to cause histone hypermethylation, which may lead to gene expression silencing ${ }^{38,39}$. We wanted to test whether hypoxia also inhibited differentiation through histone methylation. Our results indicated hypoxia increased histone methylation markers in both CHP134 and SMS-KCNR cells (Fig. 8a), which may result from the depletion of $\alpha \mathrm{KG}$ and production of $2 \mathrm{HG}$ under hypoxia (Fig. 8b). Adding a cell-permeable $\alpha \mathrm{KG}$ analog Dimethyl- $\alpha$-ketoglutarate (DMKG) reduced histone hypermethylation upon hypoxia but did not significantly increase histone acetylation (Fig. 8c). In addition, DMKG supplementation did not restore the expression of NGFR and SNCG under hypoxia (Fig. 8d). These data indicate that inhibition of histone hypermethylation is not sufficient to restore the expression of differentiation markers under hypoxia, histone acetylation might be the limiting factor for RA-induced cell differentiation under hypoxia.

\section{Discussion}

Cell differentiation is the process that a stem/progenitor cell becomes morphologically and functionally specialized. Proper cell differentiation process requires a signaling cascade to activate the transcriptional machinery and the accessibility to chromosome. Together tissuespecific differentiation markers will be expressed, and cells can differentiate. Previous studies have reported that significant metabolic reprogramming occurs during cell differentiation. Generally, undifferentiated cells primarily utilize aerobic glycolysis, but switch to oxidative phosphorylation when they are induced to differentiate ${ }^{40,41}$. The distinct metabolic phenotype between differentiated cell and undifferentiated cell indicated a critical role of mitochondrial metabolism in differentiation.

It was a century ago when Otto $\mathrm{H}$. Warburg first published his discovery that tumor cells convert large amounts of glucose to lactate through fermentation ${ }^{1}$. The Warburg effect is truly a hallmark that is identified in all kinds of cancer cells. However, the exact role of the Warburg effect in tumorigenesis has remained an enigma. The generation and excretion of lactate would 


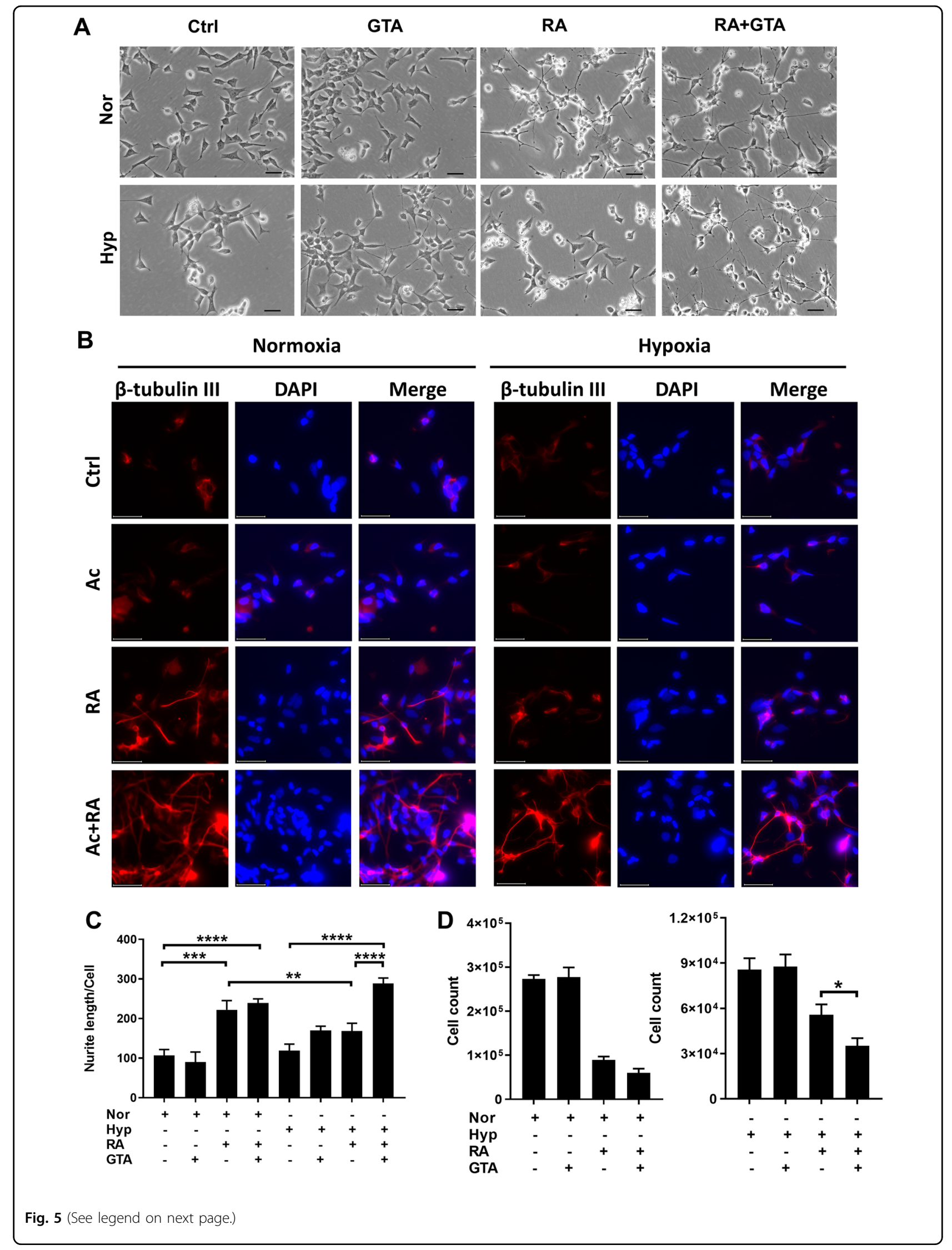


(see figure on previous page)

Fig. 5 Acetate supplementation restores neuroblastoma cell differentiation under hypoxia. a CHP134 cell differentiation induced by $10 \mu \mathrm{M}$ RA, 2 mM GTA, or $10 \mu$ M RA plus 2 mM GTA for $48 \mathrm{~h}$ under normoxia or hypoxia. Scale bar: $50 \mu \mathrm{m}$. b Immunofluorescence staining of $\beta$-tubulin III (Red) and DAPI (Blue) in CHP134 cells treated with $10 \mu \mathrm{M}$ RA, $5 \mathrm{mM}$ acetate, or $10 \mu \mathrm{M}$ RA plus $5 \mathrm{mM}$ acetate for $72 \mathrm{~h}$ under normoxia or hypoxia. Scale bar: $50 \mu \mathrm{m}$. c Quantification of neurite outgrowth in (a). d CHP134 cell proliferation measured in 12 well-plate treated with $10 \mu \mathrm{M}$ RA, 2 mM GTA, or $10 \mu \mathrm{M}$ RA plus $2 \mathrm{mM}$ GTA under normoxia or hypoxia for $48 \mathrm{~h}$. (Data in $\mathbf{c}$, $\mathbf{d}$ are represented as mean \pm SD of three biological repeats. ${ }^{*} P<0.05 ;{ }^{*} P<$ $0.01 ;{ }^{* * *} P<0.001,{ }^{* * *} P<0.0001$, determined by Student's two-tailed $t$-test.).

appear be a waste of carbon backbone and energy that is needed for proliferation. The discovery that loss or repression of mitochondrial pyruvate carrier (MPC) in cancer provided an early clue ${ }^{42}$. It has been reported that overexpression of MPC could reduce cancer cells growth and downregulates stemness markers. Oppositely, knocking out MPC or using an MPC inhibitor promotes stem cell function and organoid formation ${ }^{43}$. These data suggest that preventing pyruvate entry into mitochondria is critical for stemness maintenance. However, it's unclear how this event controls cell fate decision, and how other tumor cells without MPC downregulation maintain stemness and dedifferentiation state.

As Warburg and others pointed out, low oxygen is one way of inhibiting respiration and causing the Warburg effect $^{3,44,45}$. Hypoxia is a common feature of all solid tumors ${ }^{46}$. It has been well established that hypoxia stabilizes HIF to reprogram cellular metabolism, inhibiting mitochondrial respiration but promoting glycolysis and lactate production ${ }^{47}$. HIF-1-dependent induction of PDKs plays a key role in this metabolic reprogramming and cellular adaptation to hypoxia. While previous studies on PDKs focused on how they regulate mitochondrial activity and cell survival, here we demonstrated that induction of PDKs was also critical in regulating chromatin remodeling and cell differentiation. Treatment with PDKs inhibitor DCA restored histone acetylation under hypoxia, highlighting the potential application of DCA in combination with RA-based differentiation therapy in neuroblastoma treatment.

Many cancer cells overexpress ACSS2 to utilize environmental acetate as a fuel of biosynthesis. Acetate was reported as a nutrient source used by tumor cells for lipid synthesis and energy production upon stressed conditions $^{48-51}$. Here we demonstrated that acetate or GTA supplementation not only restored histone acetylation but also restored neuron differentiation markers expression and neuron differentiation morphology when coapplied with RA. Based on these findings, we propose that when combined with differentiation therapy, acetate supplementation may turn tumor cells' strength into a weakness, promoting differentiation in tumor cells that display a high demand for exogenous acetate. In addition to neuroblastoma, RA and other retinoids have shown promising anti-cancer effects in cell lines or preclinical models of other types of solid tumors ${ }^{52}$. It is expected that this combination therapeutic strategy might have a broad application that is not limited to neuroblastoma.

\section{Materials and methods \\ Antibodies and reagents}

The antibodies and reagents used in this study were listed in Supplemental Table S1.

\section{Cell culture and RNAi}

CHP134 and SMS-KCNR cells were obtained from Dr. John M. Maris' laboratory (Children's Hospital of Philadelphia) and Dr. C. Patrick Reynolds' laboratory (Texas Tech University Health Sciences Center $)^{53}$, respectively. Certificate of analysis is available from each group. Both cell lines were cultured in DMEM/F12 supplemented with penicillin, streptomycin, 10\% FBS. Stable CHP134 cells expressing PDK1 or PDK3 shRNA were generated through infection with lentivirus and puromycin selection. To obtain the shRNA-expressing virus, pLKOshRNA vectors (Sigma-Aldrich) were cotransfected with the third-generation lentivirus packaging plasmids (pMDLg, pCMV-VSV-G and pRsv-Rev) into HEK293T cells using FuGENE 6 Transfection Reagent (Promega). Media was changed after $24 \mathrm{~h}$ and viral supernatant was collected at $48 \mathrm{~h}$. Target cells were infected by viral supernatant (diluted 1:1 with DMEM/ F12; $6 \mu \mathrm{g} / \mathrm{ml}$ polybrene). Fresh media was added after $24 \mathrm{~h}$ and cells was selected with $2 \mu \mathrm{g} / \mathrm{ml}$ puromycin for $48 \mathrm{~h}$. Thereafter, cells were maintained in DMEM/F12 with $1 \mu \mathrm{g} / \mathrm{ml}$ puromycin.

\section{Protein isolation and western blot}

Cells were washed with PBS buffer and lysed with Harvest lysis buffer supplemented with Halt inhibitors for 5-10 min. Cell lysate was centrifuged at $5000 \mathrm{rpm}$ for 5 min at $4{ }^{\circ} \mathrm{C}$. The supernatant containing cytosolic proteins was transferred to a new EP tube. The insoluble part contains nuclear proteins and was further lysed with nuclear lysis buffer for $5 \mathrm{~min}$ at $4{ }^{\circ} \mathrm{C}$. Then the lysate was sonicated in water-bath at $4{ }^{\circ} \mathrm{C}$ for 6 cycles $(1$ cycle $=30 \mathrm{~s}$ sonication and $30 \mathrm{~s}$ cooldown). After sonication, the cell lysate was centrifuged at 15,000 rpm for $10 \mathrm{~min}$. Protein concentration was determined by BCA assay. $1 \mu \mathrm{g}$ nuclear proteins or $10 \mu \mathrm{g}$ cytosolic proteins were boiled in loading 

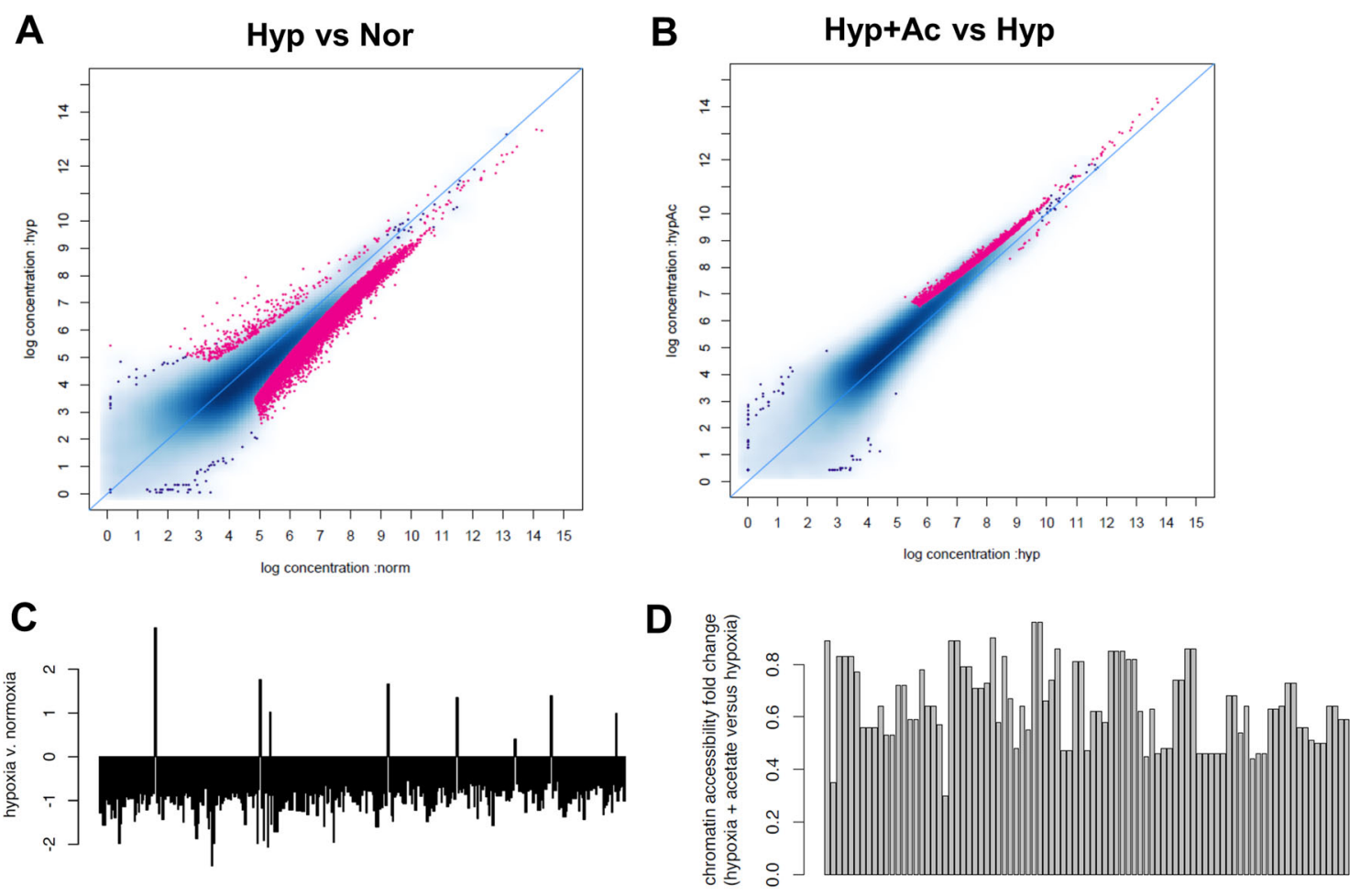

genes with RAR-RXR sites in ATAC peaks ( $n=497)$

genes with RAR-RXR sites in ATAC peaks $(n=89)$

$E$

ATAC-Seq

DEVELOPMENTAL BIOLOGY AXON GUIDANCE SIGNALLING BY NGFIMMUNE SYSTEM CELL CYCLE MITOTIC-
P75 NTR RECEPTOR MEDIATED SIGNALLING-
SIGNALING BY ERBB4SIGNALING BY ERBB4-
CELL DEATH SIGNALLING VIA NRAGE NRIF AND NADE-
MITOTIC M M G1 PHASESMITOTIC M M G1 PHASES
MYD88 MAL CASCADE INITIATED ON PLASMA MEMBRANE SEMAPHORIN INTERACTIONS SIGNALING BY ROBO RECEPTOR-

ADAPTIVE IMMUNE SYSTEM

DNA REPLICATION

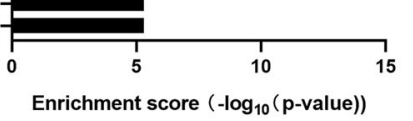

$\mathbf{F}$

Nor-Ctrl
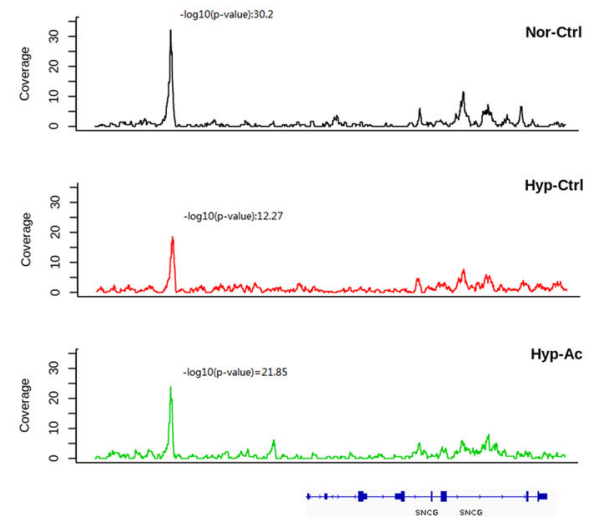

Fig. 6 (See legend on next page.) 
(see figure on previous page)

Fig. 6 ATAC-Seq reveals that chromatin accessibility of RAR-RXR target genes and differentiation markers are restored by acetate supplementation under hypoxia. a Comparison of chromatin accessibility under normoxia and hypoxia. $\mathbf{b}$ Chromatin accessibility changes in response to acetate supplementation under hypoxia. c Genes with RAR-RXR binding site showed decreased chromatin accessibility under hypoxia. d Acetate supplementation increased chromatin accessibility of genes with RAR-RXR sites under hypoxia. e Pathway enrichment of genes whose chromatin accessibility was decreased under hypoxia and restored by acetate supplementation. $\mathbf{f}$ Browser track of SNCG under normoxia, hypoxia and hypoxia with $5 \mathrm{mM}$ acetate.

A

Growth Curve

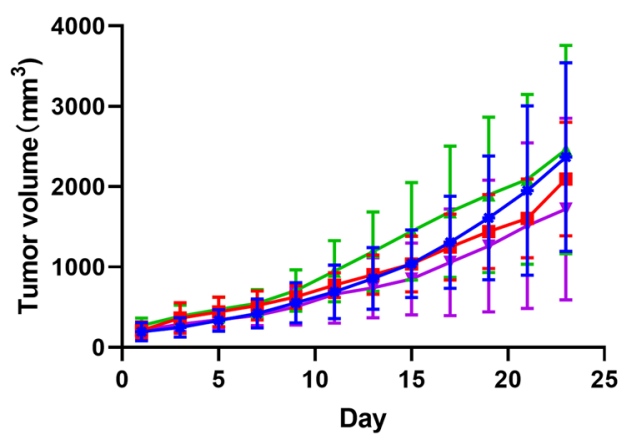

C

Body weight

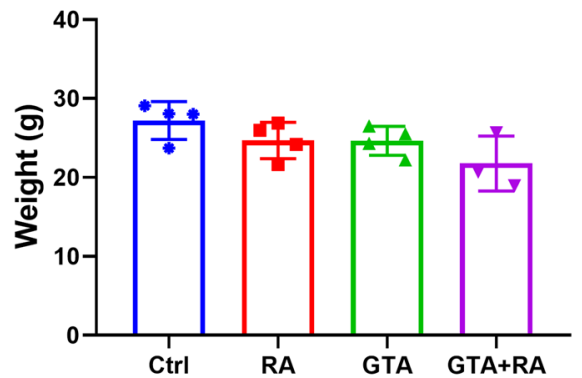

B

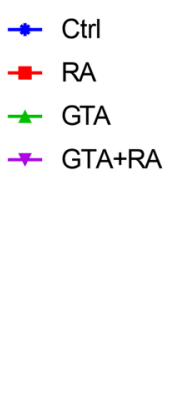

D

cis-13-Retinoic acid (Plasma)

$E$
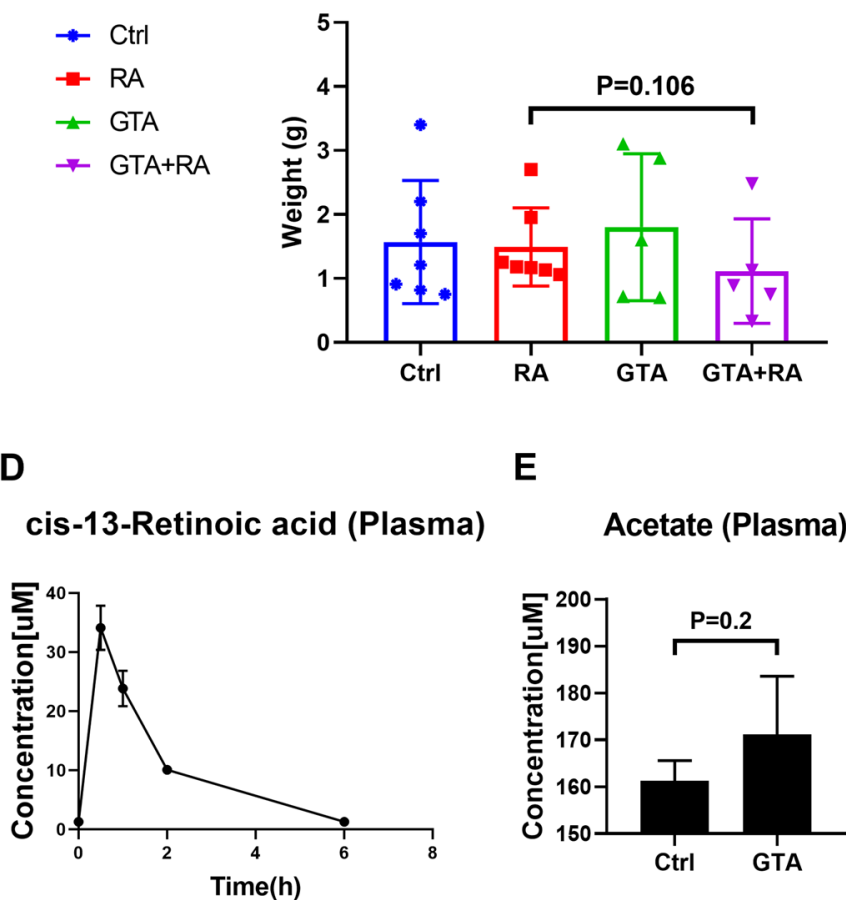

Acetate (Plasma)

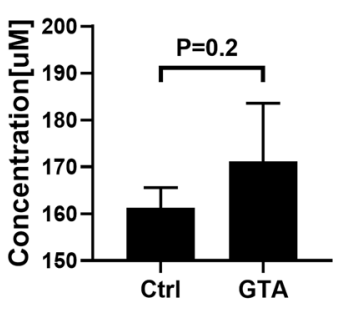

Fig. 7 In vivo xenograft study in NSG mice. a Tumor volume of CHP134 mice xenografts as measured every other day. NSG mice xenografted with CHP134 cells were randomized into 4 groups and received $10 \mathrm{mg} / \mathrm{kg}$ RA via i.p. injection, 5\% GTA in drinking water or both. b Tumor weights at experimental endpoint. (Control group: $n=7$, RA group: $n=7$, GTA group: $n=5$ and GTA + RA group: $n=5$ ). c Body weights at experimental endpoint. (Control group: $n=4$, RA group: $n=4$, GTA group: $n=4$ and GTA + RA group: $n=3$, one mouse died during treatment). d Pharmacokinetics of RA in NSG mice following administration of $10 \mathrm{mg} / \mathrm{kg}$ RA via i.p. injection. Plasma samples at each time point were collected from tail vein $(n=3)$. e Acetate measurement in the plasma of NSG mice in control and GTA group $(n=3)$. (Results in a-e are represented as mean \pm SD.).

buffer with reducing reagents, then separated with SDSPAGE. Protein were transferred onto a nitrocellulose membrane. After blocking in 5\% non-fat milk for $1 \mathrm{~h}$, the first antibody was applied. After 3x TBST washes, HRPconjugated secondary antibodies were applied. Signals were detected with an ECL kit. The complete antibody list is in Supplemental Table 1.

\section{RNA isolation, reverse transcription, and real-time PCR}

Total RNA was isolated from tissue culture plates according to the TRIzol Reagent (Invitrogen) protocol. $3 \mu \mathrm{g}$ of total RNA was used in the reverse transcription reaction using the iScript cDNA synthesis kit (Bio-Rad). Quantitative
PCR amplification was performed on the Prism 7900 Sequence Detection System (Applied Biosystems) using Taqman Gene Expression Assays (Applied Biosystems). Gene expression data were normalized to $18 \mathrm{~S}$ rRNA.

\section{Neurite outgrowth assay}

For the differentiation of neuroblastoma cells, $1 \times 10^{5}$ CHP134 or SMS-KNCR cells were plated in 6 well-plate. After overnight incubation, cells in hypoxia treatment group were pre-incubated in $0.5 \% \mathrm{O}_{2}$ hypoxia chamber for $6 \mathrm{~h}$. Then RA was added to reach a final concentration of $10 \mu \mathrm{M}$. After $48 \mathrm{~h}$ treatment, images were taken through an Olympus phase contrast microscope $(\times 20$ 


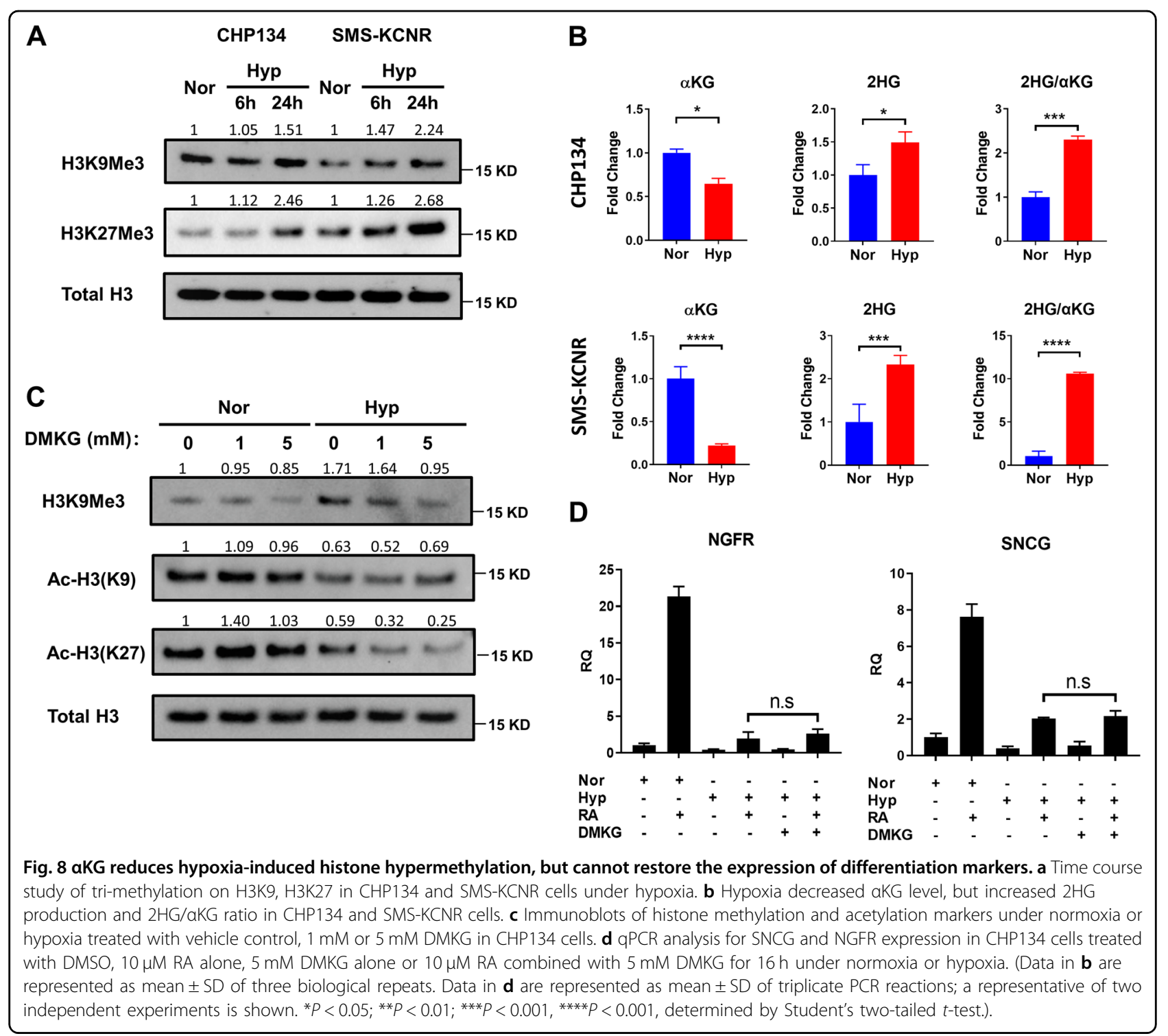

magnification). The lengths of the neurites were traced and quantified using the ImageJ plugin Neuron $J^{54}$. Within each sample, total neurite length was measured and normalized by the number of cell bodies, mean value from biological triplicates was reported.

\section{Immunofluorescence staining}

CHP134 cells were seeded into 8-champer slides with a density of 6000 cells/well and incubated with indicated treatment for 3 days. Cells were fixed with $4 \%$ PFA in $0.1 \%$ PBS-tween at room temperature for $30 \mathrm{~min}$ followed by permeabilization with $0.1 \%$ Triton $\mathrm{X}-100$ in PBS at room temperature for $10 \mathrm{~min}$. The cells were washed with PBS twice and blocked with $2.5 \%$ horse serum at room temperature for $1 \mathrm{~h}$. Then, cells were subjected to immunofluorescence staining with primary antibody overnight at
$4{ }^{\circ} \mathrm{C}$. After twice washes with PBS, cells were incubated with Alexa Fluor 594-labeled anti-rabbit secondary antibody (Life Technologies) at room temperature for $1 \mathrm{~h}$ followed by staining with DAPI for $20 \mathrm{~min}$. Images were acquired with Leica DMi8 microscope.

\section{RNA Sequencing}

The total RNA from four treatment groups (Nor_Ctrl, Nor_RA, Hyp_Ctrl and Hyp_RA, 3 replicates) was extracted using Trizol reagent according to the manufacturer's instructions $(n=3)$. The RNA-seq library was constructed and subjected to $150 \mathrm{bp}$ paired-end sequencing on an Illumina sequencing platform (Novogene). RNA-seq analysis was performed using the kallisto and sleuth analytical pipeline ${ }^{55,56}$. In brief, a transcript index was generated with reference to Ensembl version 67 for hg19. Paired-end 
mRNA-seq reads were pseudo-aligned using kallisto (v0.42.4) with respect to this transcript index using 100 bootstraps (-b 100) to estimate the variance of estimated transcript abundances. Transcript-level estimates were aggregated to transcripts per million (TPM) estimates for each gene, with gene names assigned from Ensembl using biomaRt. Differential gene expression analysis was performed using the sleuth $\mathrm{R}$ package across pairwise groups (normoxia DMSO vs. normoxia RA, normoxia DMSO vs. hypoxia DMSO, normoxia RA vs. hypoxia RA) using Wald tests, with significant hits called with a sleuth q-value $<0.05$ and fold change estimate $b>a b s(\ln (2))$.

\section{ATAC-seq}

ATAC-seq was performed and analyzed as previously described $^{57}$ (http://code.databio.org/PEPATAC/) $(n=2)$. Reads were trimmed with cutadapt version $1.8 .1^{58}$ with flags $-\mathrm{u}-50-\mathrm{U}-50-\mathrm{a}$ CTGTCTCTTATACACATCTC CGAGCCCACGAGAC -A CTGTCTCTTATACACATC TG ACGCTGCCGACGA -O 5 -m 30 -q 15. Bowtie $2^{59,60}$ version 2.3.1 was used to align trimmed reads to hg19 with flags -q --phred33 -X 2000 --fr -p 8 -x hg19, followed by samtools sort command ${ }^{61}$. Duplicates were marked with Picard-tools ${ }^{62}$ version 1.92, then samtools view with flags - b -f 1 -F12 - L were used to filter mitochondrial mapping reads with a bed file containing all chromosomes except chrM. Filtered mapping files are transferred into coverage bigwig file using deeptools with default bin size $(10 \mathrm{bps})^{63}$. SPP/phantom ${ }^{64,65}$ was run to obtain the fragment length with maximum strand cross-correlation. MACS2 callpeak function ${ }^{66}$ was then performed with flags -q 0.05 --nomodel - extsize $=1 / 2$ fragment length obtained from SPP. Associate p-values for each peak is generated and extracted from MACS2 output. Ranked gene lists were created from the RNA-Seq and ATAC-Seq datasets following differential testing. Gene set enrichment analysis were performed using the GSEA (http:// www.broadinstitute.org/gsea/index.jsp) with the C2 (CP: REACTOME) MSigDB v6.2.

\section{Cell proliferation assay}

Cell proliferation was assessed using cell counting (Beckman). Briefly, $2 \times 10^{4}$ cells were plated in 24 wellplate and attached overnight $\left(4 \times 10^{4}\right.$ cells per well if 12 well-plate was used). Then cells were treated with various conditions as indicated for $48 \mathrm{~h}(n=3)$. Before cell counting, the medium was removed and $100 \mathrm{ul} 0.25 \%$ trypsin was added to each well for $3 \mathrm{~min} .900 \mu \mathrm{l}$ medium was added to stop digestion, and $100 \mathrm{ul}$ cell suspension was transferred to cell counting vial.

\section{Metabolic tracing study}

For glucose isotope tracing, CHP134 cells were pretreated under normoxia or hypoxia for $16 \mathrm{~h}(n=3)$. Then the medium was changed to DMEM:F12 with $3.15 \mathrm{~g} / \mathrm{L}$ ${ }^{13} \mathrm{C}_{6}$-glucose with $10 \%$ dialyzed FBS for $3 \mathrm{~h}$. For acetate isotope tracing, the procedure was same as glucose tracing except that the complete DMEM:F12 medium was supplemented with $2 \mathrm{mM}{ }^{13} \mathrm{C}_{2}$-acetate.

\section{Liquid chromatography-mass spectrometry analysis}

For the metabolic tracing study, cells were washed with cold PBS, lysed in $80 \%$ Ultra LC-MS acetonitrile on ice for $15 \mathrm{~min}$, centrifuged for $10 \mathrm{~min}$ at $20,000 \times g$, and supernatant was subjected to mass spectrometry analysis. Liquid chromatography was performed using an Agilent 1290 Infinity LC system (Agilent, Santa Clara, US) coupled to a Q-TOF 6545 mass spectrometer (Agilent, Santa Clara, US). A hydrophilic interaction chromatography method (HILIC) with a BEH amide column $(100 \times 2.1 \mathrm{~mm}$ i.d., $1.7 \mu \mathrm{m}$; Waters) was used for compound separation at $35^{\circ} \mathrm{C}$ with a flow rate of $0.3 \mathrm{ml} / \mathrm{min}$. The mobile phase A consisted of $25 \mathrm{mM}$ ammonium acetate and $25 \mathrm{mM}$ ammonium hydroxide in water and mobile phase $B$ was acetonitrile. The gradient elution was $0-1 \mathrm{~min}, 85 \% \mathrm{~B}$; $1-12 \mathrm{~min}, 85 \% \mathrm{~B} \rightarrow 65 \% \mathrm{~B} ; 12-12.2 \mathrm{~min}, 65 \% \mathrm{~B} \rightarrow 40 \% \mathrm{~B}$; $12.2-15 \mathrm{~min}, 40 \% \mathrm{~B}$. After the gradient, the column was re-equilibrated at $85 \% \mathrm{~B}$ for $5 \mathrm{~min}$. The overall runtime was $20 \mathrm{~min}$, and the injection volume was $5 \mu \mathrm{L}$. Agilent Q-TOF was operated in negative mode and the relevant parameters were as listed: ion spray voltage, $3500 \mathrm{~V}$; nozzle voltage, $1000 \mathrm{~V}$; fragmentor voltage, $125 \mathrm{~V}$; drying gas flow, $11 \mathrm{~L} / \mathrm{min}$; capillary temperature, $325^{\circ} \mathrm{C}$, drying gas temperature, $350^{\circ} \mathrm{C}$; and nebulizer pressure, $40 \mathrm{psi}$. A full scan range was set at 50 to $1600(\mathrm{~m} / \mathrm{z})$. The reference mass were 119.0363 and 980.0164 . The acquisition rate was 2 spectra/s. Targeted analysis, isotopologues extraction and natural isotope abundance correction were performed by Agilent Profinder B.08.00 (Agilent Technologies).

For acetate measurement, $10 \mathrm{ul}$ of serum was mixed with $10 \mathrm{ul}$ of $50 \mathrm{uM}{ }^{13} \mathrm{C}_{2}$-acetate. Then, the mixture was precipitated by the addition of $80 \mathrm{ul}$ acetonitrile and centrifuged for $10 \mathrm{~min}$ at $20,000 \times g .80 \mathrm{ul}$ supernatant was transferred to a new tube and derivertized according to the published protocol ${ }^{67}$. $2 \mathrm{ul}$ sample was subjected to mass spectrometry analysis using Agilent 1290 coupled to Q-TOF 6545. ZORBAX Eclipse Plus C18 columns (50 × $2.1 \mathrm{~mm}, 1.8 \mu \mathrm{m}$, Agilent) was used for compound separation at $35^{\circ} \mathrm{C}$ with a flow rate of $0.3 \mathrm{ml} / \mathrm{min}$. The mobile phase were $5 \mathrm{mM}$ ammonium formate and $0.1 \%$ formic acid in water (A) and $90 \%$ acetonitrile (B). The gradient elution was $0-0.5 \mathrm{~min}, 10 \% \mathrm{~B} ; 0.5-4 \mathrm{~min}, 10 \%$ $\mathrm{B} \rightarrow 90 \% \mathrm{~B} ; 4-7.5 \mathrm{~min}, 90 \% \mathrm{~B}$. After the gradient, the column was re-equilibrated at $10 \% \mathrm{~B}$ for $2.5 \mathrm{~min}$. Agilent Q-TOF was operated in positive mode with ion spray voltage at $3500 \mathrm{~V}$. The ratio of acetate to ${ }^{13} \mathrm{C}_{2}$-acetate was calculated using Agilent Profinder software B.08.00. 
For 13-cis-retinoic acid measurement, serum samples were collected at $0.5,1,2$ and $6 \mathrm{~h}$ after RA injection $(10 \mathrm{mg} / \mathrm{kg}$, i.p.). $10 \mathrm{ul}$ serum was mixed with $90 \mathrm{ul}$ extraction buffer (ethyl acetate:n-hexane:IPA, 30:60:10). After $20 \mathrm{~min}$ on a shaker at $120 \mathrm{rpm}$, the mixture was centrifuged at $5000 \mathrm{~g}$ for $5 \mathrm{~min}$. The organic layer was collected into a new tube and dried using speed vacuum. The residue was reconstituted with $100 \mathrm{ul}$ of $5 \mathrm{mM}$ ammonium formate in water/acetonitrile (1:1). $5 \mathrm{ul} \mathrm{sam-}$ ple was subjected to mass spectrometry analysis using Agilent 1290 coupled to QQQ 6470. ZORBAX Eclipse Plus C18 columns $(50 \times 2.1 \mathrm{~mm}, 1.8 \mu \mathrm{m}$, Agilent $)$ was used for compound separation at $35^{\circ} \mathrm{C}$ with a flow rate of $0.3 \mathrm{ml} / \mathrm{min}$. The mobile phase were $5 \mathrm{mM}$ ammonium formate and $0.1 \%$ formic acid in water (A) and $90 \%$ acetonitrile (B). The gradient elution was $0-0.5 \mathrm{~min}, 50 \%$ B; $0.5-2$ min, $50 \%$ B $\rightarrow 90 \%$ B; $2-4.8$ min, $90 \%$ B. After the gradient, the column was re-equilibrated at $50 \% \mathrm{~B}$ for $2.2 \mathrm{~min}$. Agilent QQQ 6470 was operated in positive mode with ion spray voltage at $3000 \mathrm{~V}$. The MRM transitions were $301.2 \rightarrow 159.1 / 205.1$. Data was processed by Agilent MassHunter Quantitative Analysis for QQQ B.07.01. (Agilent Technologies)

\section{Mouse xenograft model}

In vivo studies were performed in accordance with protocols approved by the Institutional Animal Care and Use Committee at Stanford University and in compliance with all regulatory standards. 6 to 8 -week-old male NSG mice were provided by Dr. Erinn Rankin. $2 \times 10^{6}$ CHP134 cells suspended in $100 \mu \mathrm{L}$ of RPMI-1640 with $50 \%$ Matrigel (BD Biosciences) were implanted subcutaneously into the dorsal flank of the mice. Tumor growth was recorded using digital caliper every other day, and tumor volumes were estimated using the formula: $\left(\mathrm{L} \times \mathrm{W}^{2}\right) / 2$, where $\mathrm{L}=$ length and $\mathrm{W}=$ width of tumor. The animals were randomized into 4 groups (Control, RA, GTA and $\mathrm{GTA}+\mathrm{RA}$ ) when the tumor volumes reached approximately $150 \mathrm{~mm}^{3} .10 \mathrm{mg} / \mathrm{kg}$ RA or DMSO vehicle was injected intraperitoneally daily for 3 weeks. Mice received GTA in the drinking water ( $5 \%$ by weight).

\section{Statistics}

For cell proliferation and MS experiments, three biological repeats were used for data analysis. Results were represented as mean \pm SD of three biological repeats. The Student's t-test will be performed to determine the significance between groups (two-tailed, unequal variance).

\section{Acknowledgements}

This work was supported by a NIH R00 Grant (CA184239) and a Stanford Child Health Research Institute and the Stanford NIHNCATS-CTSA (UL1 TR001085) Pilot Early Career Award to J.Y., and by P50-HG007735 and R35-CA209919 (to H. Y.C.). H.Y.C. is an Investigator of the Howard Hughes Medical Institute. We thank Dr. Curt R. Fischer (Stanford ChEM-H Institute) for the support and facilities. We thank Dr. Beverly S. Mitchell (Stanford Cancer Institute) for critically reviewing the paper and helpful comments.

\section{Author details}

'Department of Radiation Oncology, Stanford University School of Medicine, Stanford, CA 94305, USA. ²Department of Genetics, Stanford University School of Medicine, Stanford, CA 94305, USA. ${ }^{3}$ Center for Personal Dynamic

Regulomes, Stanford University School of Medicine, Stanford, CA 94305, USA. ${ }^{4}$ Olivia Consulting Service, Redwood City, CA 94063, USA. ${ }^{5}$ Division of Oncology and Center for Childhood Cancer Research, Children's Hospital of Philadelphia and the Perelman School of Medicine at the University of Pennsylvania, Philadelphia, PA 19104, USA. ${ }^{6}$ Howard Hughes Medical Institute, Stanford University, Stanford, CA 94305, USA

\section{Data availability}

RNA-Seq data are available on NCBI Sequence Read Archive (BioProject Accession No.: PRJNA596588). ATAC-Seq data are available on NCBI Sequence Read Archive (BioProject Accession No.: PRJNA596881).

\section{Conflict of interest}

Y.L and J.Y have filed a provisional patent application: Treatment of cancer with combination of agents.

\section{Publisher's note}

Springer Nature remains neutral with regard to jurisdictional claims in published maps and institutional affiliations.

Supplementary Information accompanies this paper at (https://doi.org/ 10.1038/s41419-020-2303-9).

Received: 23 September 2019 Revised: 23 January 2020 Accepted: 23 January 2020

Published online: 06 February 2020

\section{References}

1. Warburg, O., Posener, K. \& Negelein, E. On the metabolism of carcinoma cells. Biochem Z. 152, 309-344 (1924).

2. Warburg, O., Wind, F. \& Negelein, E. The metabolism of tumors in the body. J. Gen. Physiol. 8, 519-530 (1927)

3. Warburg, O. On the origin of cancer cells. Science 123, 309-314 (1956).

4. Jogi, A. et al. Hypoxia alters gene expression in human neuroblastoma cells toward an immature and neural crest-like phenotype. Proc. Natl Acad. Sci. USA 99, 7021-7026 (2002).

5. Fredlund, E., Ringner, M., Maris, J. M. \& Pahlman, S. High Myc pathway activity and low stage of neuronal differentiation associate with poor outcome in neuroblastoma. Proc. Natl Acad. Sci. USA 105, 14094-14099 (2008).

6. Pahlman, S. \& Mohlin, S. Hypoxia and hypoxia-inducible factors in neuroblastoma. Cell tissue Res. 372, 269-275 (2018).

7. Jogi, A. et al. Hypoxia-induced dedifferentiation in neuroblastoma cells. Cancer Lett. 197, 145-150 (2003).

8. van Groningen, T. et al. Neuroblastoma is composed of two super-enhancerassociated differentiation states. Nat. Genet 49, 1261-1266 (2017).

9. Colon, N. C. \& Chung, D. H. Neuroblastoma. Adv. Pediatr. 58, $297-311$ (2011).

10. Durinck, K. \& Speleman, F. Epigenetic regulation of neuroblastoma development. Cell tissue Res. 372, 309-324 (2018).

11. Pugh, T. J. et al. The genetic landscape of high-risk neuroblastoma. Nat. Genet 45, 279-284 (2013)

12. Evans, A. E., Gerson, J. \& Schnaufer, L. Spontaneous regression of neuroblastoma. Natl Cancer Inst. Monogr. 44, 49-54 (1976).

13. Hero, B. et al. Localized infant neuroblastomas often show spontaneous regression: results of the prospective trials NB95-S and NB97. J. Clin. Oncol. 26, 1504-1510 (2008).

14. Reynolds, C. P., Matthay, K. K., Villablanca, J. G. \& Maurer, B. J. Retinoid therapy of high-risk neuroblastoma. Cancer Lett. 197, 185-192 (2003). 
15. Sidell, N. Retinoic acid-induced growth inhibition and morphologic differentiation of human neuroblastoma cells in vitro. J. Natl Cancer Inst. 68 , 589-596 (1982).

16. Matthay, K. K. et al. Treatment of high-risk neuroblastoma with intensive chemotherapy, radiotherapy, autologous bone marrow transplantation, and 13-cis-retinoic acid. Children's Cancer Group. N. Engl. J. Med 341, 1165-1173 (1999).

17. Huang, M. E. et al. Use of all-trans retinoic acid in the treatment of acute promyelocytic leukemia. Blood 72, 567-572 (1988).

18. Rao, Y., Li, R. H. \& Zhang, D. Q. A drug from poison: how the therapeutic effect of arsenic trioxide on acute promyelocytic leukemia was discovered. Sci. China Life Sci. 56, 495-502 (2013).

19. Chlapek, P., Slavikova, V., Mazanek, P., Sterba, J. \& Veselska, R. Why differentiation therapy sometimes fails: molecular mechanisms of resistance to retinoids. Int J. Mol. Sci. https://doi.org/10.3390/ijms19010132 (2018).

20. Dzieran, J. et al. MYCN-amplified neuroblastoma maintains an aggressive and undifferentiated phenotype by deregulation of estrogen and NGF signaling. Proc. Natl Acad. Sci. USA 115, E1229-E1238 (2018).

21. Buchman, V. L., Adu, J., Pinon, L. G., Ninkina, N. N. \& Davies, A. M. Persyn, a member of the synuclein family, influences neurofilament network integrity. Nat. Neurosci. 1, 101-103 (1998).

22. Koster, J., Molenaar, J. J. \& Versteeg, R. R2: Accessible web-based genomics analysis and visualization platform for biomedical researchers. Cancer Res. https://doi.org/10.1158/1538-7445.Compsysbio-B1-05 (2015).

23. Korzus, E. et al. Transcription factor-specific requirements for coactivators and their acetyltransferase functions. Science 279, 703-707 (1998).

24. Dilworth, F. J., Fromental-Ramain, C., Yamamoto, K. \& Chambon, P. ATP-driven chromatin remodeling activity and histone acetyltransferases act sequentially during transactivation by RAR/RXR in vitro. Mol. Cell 6, 1049-1058 (2000).

25. Wellen, K. E. et al. ATP-citrate lyase links cellular metabolism to histone acetylation. Science 324, 1076-1080 (2009).

26. Kim, J. W., Tchernyshyov, I., Semenza, G. L. \& Dang, C. V. HIF-1-mediated expression of pyruvate dehydrogenase kinase: a metabolic switch required for cellular adaptation to hypoxia. Cell Metab. 3, 177-185 (2006).

27. Papandreou, I., Cairns, R. A., Fontana, L., Lim, A. L. \& Denko, N. C. HIF-1 mediates adaptation to hypoxia by actively downregulating mitochondrial oxygen consumption. Cell Metab. 3, 187-197 (2006).

28. Lu, C. W., Lin, S. C., Chen, K. F., Lai, Y. Y. \& Tsai, S. J. Induction of pyruvate dehydrogenase kinase-3 by hypoxia-inducible factor- 1 promotes metabolic switch and drug resistance. J. Biol. Chem. 283, 28106-28114 (2008).

29. Zhang, $C$. et al. Biological significance of the suppression of oxidative phosphorylation in induced pluripotent stem cells. Cell Rep. 21 2058-2065 (2017).

30. Bulusu, V. et al. Acetate recapturing by nuclear acetyl-CoA synthetase 2 prevents loss of histone acetylation during oxygen and serum limitation. Cell Rep. 18, 647-658 (2017)

31. Kinnaird, A., Zhao, S., Wellen, K. E. \& Michelakis, E. D. Metabolic control of epigenetics in cancer. Nat. Rev. Cancer 16, 694-707 (2016).

32. Mews, P. et al. Acetyl-CoA synthetase regulates histone acetylation and hippocampal memory. Nature 546, 381-386 (2017).

33. Yoshii, Y. et al. Cytosolic acetyl-CoA synthetase affected tumor cell survival under hypoxia: the possible function in tumor acetyl-CoAvacetate metabolism. Cancer Sci. 100, 821-827 (2009).

34. Buckley, B. M. \& Williamson, D. H. Origins of blood acetate in the rat. Biochem J. 166, 539-545 (1977).

35. Dang, L. et al. Cancer-associated IDH1 mutations produce 2-hydroxyglutarate. Nature 462, 739-744 (2009).

36. Scourzic, L., Mouly, E. \& Bernard, O. A. TET proteins and the control of cytosine demethylation in cancer. Genome Med. 7, 9 (2015).

37. $\mathrm{Xu}, \mathrm{W}$. et al. Oncometabolite 2-hydroxyglutarate is a competitive inhibitor of alpha-ketoglutarate-dependent dioxygenases. Cancer Cell 19, 17-30 (2011).

38. Intlekofer, A. M. et al. Hypoxia Induces Production of L-2-Hydroxyglutarate. Cell Metab. 22, 304-311 (2015)

39. Oldham, W. M., Clish, C. B., Yang, Y. \& Loscalzo, J. Hypoxia-Mediated Increases in L-2-hydroxyglutarate coordinate the metabolic response to reductive stress. Cell Metab. 22, 291-303 (2015).
40. Riester, M. et al. The Warburg effect: persistence of stem-cell metabolism in cancers as a failure of differentiation. Ann. Oncol. 29, 264-270 (2018).

41. Zheng, $X$. et al. Metabolic reprogramming during neuronal differentiation from aerobic glycolysis to neuronal oxidative phosphorylation. elife https://doi. org/10.7554/eLife.13374 (2016).

42. Schell, J. C. et al. A role for the mitochondrial pyruvate carrier as a repressor of the warburg effect and colon cancer cell growth. Mol. Cell 56, 400-413 (2014).

43. Schell, J. C. et al. Control of intestinal stem cell function and proliferation by mitochondrial pyruvate metabolism. Nat. Cell Biol. 19, 1027 (2017).

44. Kim, J. W. \& Dang, C. V. Cancer's molecular sweet tooth and the Warburg effect. Cancer Res 66, 8927-8930 (2006)

45. Xie, H. \& Simon, M. C. Oxygen availability and metabolic reprogramming in cancer. J. Biol. Chem. 292, 16825-16832 (2017).

46. Yun, Z. \& Giaccia, A. J. Tumor deprivation of oxygen and tumor suppressor gene function. Methods Mol. Biol. 223, 485-504 (2003).

47. Hubbi, M. E. \& Semenza, G. L. Regulation of cell proliferation by hypoxiainducible factors. Am. J. Physiol. Cell Physiol. 309, C775-C782 (2015).

48. Comerford, S. A. et al. Acetate dependence of tumors. Cell 159, 1591-1602 (2014).

49. Mashimo, T. et al. Acetate is a bioenergetic substrate for human glioblastoma and brain metastases. Cell 159, 1603-1614 (2014).

50. Schug, Z. T. et al. Acetyl-CoA synthetase 2 promotes acetate utilization and maintains cancer cell growth under metabolic stress. Cancer Cell 27, 57-71 (2015).

51. Gao, X. et al. Acetate functions as an epigenetic metabolite to promote lipid synthesis under hypoxia. Nat. Commun. 7, 11960 (2016).

52. Altucci, L., Leibowitz, M. D., Ogilvie, K. M., de Lera, A. R. \& Gronemeyer, H. RAR and RXR modulation in cancer and metabolic disease. Nat. Rev. Drug Disco. 6 793-810 (2007).

53. Reynolds, C. P. et al. Characterization of Human Neuroblastoma CellLines Established before and after Therapy. J. Nat/ Cancer / 76, 375-387 (1986).

54. Meijering, E. et al. Design and validation of a tool for neurite tracing and analysis in fluorescence microscopy images. Cytom. A 58, 167-176 (2004).

55. Bray, N. L., Pimentel, H., Melsted, P. \& Pachter, L. Near-optimal probabilistic RNA-seq quantification. Nat. Biotechnol. 34, 525-527 (2016).

56. Pimentel, H., Bray, N. L., Puente, S., Melsted, P. \& Pachter, L. Differential analysis of RNA-seq incorporating quantification uncertainty. Nat. Methods 14, 687-690 (2017)

57. Corces, M. R. et al. An improved ATAC-seq protocol reduces background and enables interrogation of frozen tissues. Nat. Methods 14, 959-962 (2017).

58. Martin, M. Cutadapt removes adapter sequences from high-throughput sequencing reads. EMBnet. J. 17, 10-12 (2011).

59. Langmead, B., Trapnell, C., Pop, M. \& Salzberg, S. L. Ultrafast and memoryefficient alignment of short DNA sequences to the human genome. Genome Biol. 10, R25 (2009).

60. Langmead, B. \& Salzberg, S. L. Fast gapped-read alignment with Bowtie 2. Nat methods 9, 357-359 (2012).

61. Li, H. et al. The Sequence Alignment/Map format and SAMtools. Bioinformatics 25, 2078-2079 (2009)

62. Picard-tools. http://broadinstitute.github.io/picard.

63. Ramirez, F. et al. deepTools2: a next generation web server for deepsequencing data analysis. Nucleic Acids Res. 44, W160-W165 (2016).

64. Landt, S. G. et al. ChIP-seq guidelines and practices of the ENCODE and modENCODE consortia. Genome Res. 22, 1813-1831 (2012)

65. Kharchenko, P. V., Tolstorukov, M. Y. \& Park, P. J. Design and analysis of ChIPseq experiments for DNA-binding proteins. Nat. Biotechnol. 26, 1351-1359 (2008).

66. Zhang, Y. et al. Model-based analysis of ChIP-Seq (MACS). Genome Biol. 9, R137 (2008).

67. Tan, B., Lu, Z., Dong, S., Zhao, G. \& Kuo, M. S. Derivatization of the tricarboxylic acid intermediates with O-benzylhydroxylamine for liquid chromatography-tandem mass spectrometry detection. Anal. Biochem. 465, 134-147 (2014). 